\title{
QCD SELECTION RULES IN POLARIZED HADRON COLLISIONS*
}

\author{
R. L. Jaffe \\ Center for Theoretical Physics, Laboratory for Nuclear Science \\ and Department of Physics \\ Massachusetts Institute of Technology, Cambridge, Massachusetts 02139 \\ and \\ Lyman Physics Laboratory, Harvard University \\ Cambridge, Massachusetts 02138 \\ and \\ N. Saito \\ Radiation Laboratory \\ RIKEN (The Institute of Physical and Chemical Research) \\ Wako, Saitama, 351-01, Japan
}

(MIT-CTP-2519 HUTP-95/A013 Submitted to Physics Letters B March 1996)

\begin{abstract}
Plans are underway to measure spin asymmetries at large momentum transfer in hadron hadron collisions at RHIC and elsewhere. Proposals have focused on measuring quark transversity and quark and gluon helicity distributions in the nucleon. These experiments will also provide a strong and simple test of perturbative QCD, namely that $\mathcal{A}_{T T} / \mathcal{A}_{L L} \ll 1$ in $p p \rightarrow 2$ jets $+X$ and several related processes, whereas $\mathcal{A}_{T T} / \mathcal{A}_{L L} \sim 1$ in Drell-Yan production of muon pairs. The prediction tests the helicity, twist and chirality selection rules of perturbative QCD that form the foundation for the analysis of spin dependent hard processes. We estimate the ratio $\mathcal{A}_{T T} / \mathcal{A}_{L L}$ numerically for polarized protons at RHIC.
\end{abstract}

\footnotetext{
*This work is supported in part by funds provided by the U.S. Department of Energy (D.O.E.) under cooperative agreement \#DF-FC02-94ER40818 and \#DE-FG02-92ER40702 and in part by funds provided by the National Science Foundation under grant \# PHY 92-18167.
} 
Gluons are abundant in the nucleon. In addition their color charge is larger than that of quark and antiquarks. So in general gluon-gluon $(g g)$ and gluon-quark $(g q)$ scattering dominate hard processes unless forbidden by initial or final state specifications. Thus, for example, $g g, g q$ scattering dominate two jet $(j j)$ production in $p p$ collisions except at large $x_{1}$ and $x_{2}$ where valence quarks overwhelm gluons. As an example where selection of a final state removes gluons from consideration one need look no further than the classic Drell-Yan process at leading order in $\alpha_{s}$, since production of lepton pairs requires $q \bar{q}$ annihilation. As a result $p p \rightarrow \ell \bar{\ell} X$ is considerably suppressed compared to $p p \rightarrow j j X$ for comparable kinematics (trivial factors of $\alpha_{s}$ and $\alpha_{E M}$ aside).

The possibility that polarized gluons are abundant in a polarized nucleon is now taken quite seriously. It therefore seems appropriate to look at the relative magnitude of spin dependent hard processes depending whether polarized gluons can contribute. The helicity and chirality structure of the leading-twist quark and gluon distributions have a dramatic effect on these considerations. The helicity difference gluon distribution, $\Delta g\left(x, Q^{2}\right)$, is of considerable interest in connection with the nucleon spin problem and may be large. [1] However, there is no helicity flip gluon distribution at leading twist and therefore gluons do not contribute to transverse asymmetries at leading twist since transverse asymmetries originate from the helicity flip amplitude. As a result there is a striking difference between the transverse asymmetry, $\mathcal{A}_{T T}$ and the longitudinal asymmetry, $\mathcal{A}_{L L}$ in processes dominated by $g g$ and/or $g q$ scattering. In contrast, processes like Drell-Yan where gluons do not contribute, show no dramatic difference between $\mathcal{A}_{T T}$ and $\mathcal{A}_{L L}$.

In this Letter we review the helicity and chirality assignments of quark and gluon distributions at leading twist and derive the "selection rules" that are our principle results. Next we briefly review earlier work and explain what we have added to the subject. Finally we take some reasonable models of polarized quark and gluon distributions in the nucleon and use PYTHIA, one of the widely-used QCD event generators, to make numerical estimates for a future polarized proton-proton collider environment at RHIC.

\section{PARTON DISTRIBUTION FUNCTIONS AND SELECTION RULES IN POLARIZED HADRON SCATTERING}

The helicity, chirality and transversity classification of parton distributions within a spin1/2 target are now well understood through twist-three. [2] Dominant effects are determined by twist-two distributions which scale (modulo logarithms) in the deep inelastic limit. Subdominant effects, which are suppressed by a power of a large momentum scale, are described by twist-three distributions.

The twist-two quark distributions are $q\left(x, Q^{2}\right), \Delta q\left(x, Q^{2}\right)$, and $\delta q\left(x, Q^{2}\right)$,

- $q^{a}\left(x, Q^{2}\right)$ is the familiar spin averaged quark distribution, which preserves quark chirality. $a$ is a flavor label.

- $\Delta q^{a}\left(x, Q^{2}\right)$ is the helicity difference quark distribution. It measures the probability to find a quark with helicity parallel minus antiparallel to the target helicity. $\Delta q$ also preserves quark chirality. 


\begin{tabular}{|l|l|ccc|}
\hline \hline & Helicity & Average & Difference & Flip \\
\hline \multirow{3}{*}{ Quark } & Notation & $q\left(x, Q^{2}\right)$ & $\Delta q\left(x, Q^{2}\right)$ & $\delta q\left(x, Q^{2}\right)$ \\
& Helicity amplitudes & $A_{\frac{1}{2} \frac{1}{2}, \frac{1}{2} \frac{1}{2}}+A_{\frac{1}{2}-\frac{1}{2}, \frac{1}{2}-\frac{1}{2}}$ & $A_{\frac{1}{2} \frac{1}{2}, \frac{1}{2} \frac{1}{2}-A_{\frac{1}{2}-\frac{1}{2}, \frac{1}{2}-\frac{1}{2}}}$ & $A_{-\frac{1}{2} \frac{1}{2}, \frac{1}{2}-\frac{1}{2}}$ \\
& Chirality & even & even & - \\
\hline \multirow{3}{*}{ Gluon } & Notation & $g\left(x, Q^{2}\right)$ & $\Delta g\left(x, Q^{2}\right)$ & - \\
& Helicity Amplitudes & $A_{\frac{1}{2} 1, \frac{1}{2} 1}+A_{\frac{1}{2}-1, \frac{1}{2}-1}$ & $A_{\frac{1}{2} 1, \frac{1}{2} 1}-A_{\frac{1}{2}-1, \frac{1}{2}-1}$ & - \\
& Chirality & even & even & - \\
\hline \hline
\end{tabular}

TABLE I. Twist-two quark and gluon distribution in the nucleon classified according to target helicity and quark helicity. Helicity amplitudes for virtual quark/nucleon forward scattering are denoted $A_{\Lambda \lambda, \Lambda^{\prime} \lambda^{\prime}}$. Distributions are classified according to whether quark chirality is conserved (even) or not (odd) in the quark/nucleon amplitude.

- $\delta q^{a}\left(x, Q^{2}\right)$ is the transversity difference quark distribution. It measures the probability to find a quark polarized parallel minus antiparallel to the polarization of a target polarized transverse to its (infinite) momentum. In a helicity basis, $\delta q^{a}$ is off-diagonal in the helicity of both the quark and target. The transverse eigenstates can be written as superpositions of helicity eigenstates - $|\top, \perp\rangle=\frac{1}{\sqrt{2}}(|+\rangle \pm|-\rangle)$ In the squared amplitude, the difference of $T \top$ and $T \perp$ isolates helicity flip in the helicity basis. The quark helicity changes by $\Delta \lambda= \pm 1$ and the target helicity changes by $\Delta \Lambda=\mp 1$. $\delta q^{a}$ flips chirality as well as helicity and therefore decouples from deep inelastic lepton scattering.

The gluon distributions at twist-two have the same structure $\left(g\left(x, Q^{2}\right)\right.$ and $\left.\Delta g\left(x, Q^{2}\right)\right)$ with the striking exception that there is no gluon distribution analogous to $\delta q$. Because the independent gluon states have helicity $\lambda= \pm 1$, a helicity flip gluon distribution would require the target to absorb $\Delta \Lambda=\mp 2$, which is forbidden for a spin- $\frac{1}{2}$ target. A target with spin greater than $\frac{1}{2}$ can have a helicity flip gluon distribution in leading twist. It is known as $\Delta\left(x, Q^{2}\right)$ and some of its properties are explored in Refs. [3]. Also, there is a helicity flip gluon distribution at twist-three where $\lambda=0$ can appear, but its effects are suppressed by a power of a large momentum scale in hard processes. The properties of these distributions are summarized in Table I.

As a first example we consider $p p \rightarrow j j X$ at energies and momentum transfers in the perturbative QCD regime. As usual, the cross section can be written as the product of parton distribution functions and parton-parton hard scattering cross sections. The parton distributions must be chosen according to the polarization of the initial hadrons. The longitudinal asymmetry, $\mathcal{A}_{L L}$ receives contributions from polarized gluons through $\Delta g$, and polarized quarks (or antiquarks) through $\Delta q(\Delta \bar{q})$.

$$
\mathcal{A}_{L L}^{j j}=\frac{[\Delta g \otimes \Delta g] \Delta \sigma_{g g}^{j j}+[\Delta g \otimes \Delta q] \Delta \sigma_{g q}^{j j}+[\Delta q \otimes \Delta q] \Delta \sigma_{q q}^{j j}+[\Delta q \otimes \Delta \bar{q}] \Delta \sigma_{q \bar{q}}^{j j}+\ldots}{[g \otimes g] \sigma_{g g}^{j j}+[g \otimes q] \sigma_{g q}^{j j}+[q \otimes q] \sigma_{q q}^{j j}+[q \otimes \bar{q}] \sigma_{q \bar{q}}^{j j}+\ldots} .
$$

in an obvious notation. The terms denoted by ... include anti-quark gluon and anti-quark anti-quark scattering. 
In contrast the transverse asymmetry receives contribution only from $q q$, $q \bar{q}$ and $\bar{q} \bar{q}$ because there is no helicity flip gluon distribution. Furthermore the requirement of helicity conservation in hard scattering requires $q q$ or $\bar{q} \bar{q}$ scattering to proceed via an interference between direct and exchange graphs as shown in Figure 1, reducing the contribution to the cross section by a factor of $\sim \frac{1}{11}$. [4, 边. The net result is

$$
\mathcal{A}_{T T}^{j j}=\frac{[\delta q \otimes \delta q] \delta \sigma_{q q}^{j j}+[\delta q \otimes \delta \bar{q}] \delta \sigma_{q \bar{q}}^{j j}+[\delta \bar{q} \otimes \delta \bar{q}] \delta \sigma_{\bar{q}}^{j j}}{[g \otimes g] \sigma_{g g}^{j j}+[g \otimes q] \sigma_{g q}^{j j}+[q \otimes q] \sigma_{q q}^{j j}+[q \otimes \bar{q}] \sigma_{q \bar{q}}^{j j}+\ldots} .
$$

where the gluons are absent and the $q q$ and $\bar{q} \bar{q}$ contributions are anomalously small. So the ratio of transverse to longitudinal asymmetries is given by

$$
\frac{\mathcal{A}_{T T}^{j j}}{\mathcal{A}_{L L}^{j j}}=\frac{[\delta q \otimes \delta q] \delta \sigma_{q q}^{j j}+[\delta \bar{q} \otimes \delta \bar{q}] \delta \sigma_{\bar{q} \bar{q}}^{j j}+[\delta q \otimes \delta \bar{q}] \delta \sigma_{q \bar{q}}^{j j}}{[\Delta g \otimes \Delta g] \Delta \sigma_{g g}^{j j}+[\Delta g \otimes \Delta q] \Delta \sigma_{g q}^{j j}+[\Delta q \otimes \Delta q] \Delta \sigma_{q q}^{j j}+[\Delta q \otimes \Delta \bar{q}] \Delta \sigma_{q \bar{q}}^{j j}+\ldots},
$$

For convenience the $\sigma^{j j}, \Delta \sigma^{j j}$ and $\delta \sigma^{j j}$ are given in Table II. He found the helicity amplitude

\begin{tabular}{|c|c|c|c|}
\hline $\begin{array}{c}\text { Parton process } \\
a b \rightarrow c d\end{array}$ & $\begin{array}{c}\text { Spin Average } \\
\text { Cross Section }-\sigma_{a b}^{c d}\end{array}$ & $\begin{array}{c}\text { Helicity Dependent } \\
\text { Cross Section }-\Delta \sigma_{a b}^{c d}\end{array}$ & $\begin{array}{l}\text { Transversity Dependent } \\
\text { Cross Section }-\delta \sigma_{a b}^{c d}\end{array}$ \\
\hline $\begin{array}{c}q \bar{q} \rightarrow \gamma^{*} \rightarrow \ell \bar{\ell} \\
q q \rightarrow q q \\
q q^{\prime} \rightarrow q q^{\prime} \\
q \bar{q} \rightarrow q \bar{q} \\
q \bar{q} \rightarrow q^{\prime} \bar{q}^{\prime} \\
q \bar{q}^{\prime} \rightarrow q \bar{q}^{\prime} \\
q \bar{q} \rightarrow g g \\
q \bar{q} \rightarrow g \gamma\end{array}$ & $\begin{array}{c}\frac{\hat{u}^{2}+\hat{t}^{2}}{\hat{s}^{2}} \\
\frac{\hat{s}^{2}+\hat{u}^{2}}{\hat{t}^{2}}+\frac{\hat{s}^{2}+\hat{t}^{2}}{\hat{u}^{2}}-\frac{2}{3} \frac{\hat{s}^{2}}{\hat{u} \hat{t}} \\
\frac{\hat{s}^{2}+\hat{u}^{2}}{\hat{t}^{2}} \\
\frac{\hat{s}^{2}+\hat{u}^{2}}{\hat{t}^{2}}+\frac{\hat{u}^{2} \hat{t}^{2}}{\hat{t}^{2}}-\frac{2}{3} \frac{\hat{u}^{2}}{\hat{s} \hat{t}} \\
\frac{\hat{u}^{2}+\hat{t}^{2}}{\hat{s}^{2}} \\
\frac{\hat{s}^{2}+\hat{u}^{2}}{\hat{t}^{2}} \\
\frac{8}{3} \frac{\hat{t}^{2}+\hat{u}^{2}}{\hat{t} \hat{u}}-6 \frac{\hat{t}^{2}+\hat{u}^{2}}{\hat{s}^{2}} \\
\frac{\hat{t}^{2}+\hat{u}^{2}}{\hat{t} \hat{u}}\end{array}$ & $\begin{array}{c}-\frac{\hat{t}^{2}+\hat{t}^{2}}{\hat{t}^{2}} \\
\frac{\hat{s}^{2}-\hat{u}^{2}}{\hat{t}^{2}}+\frac{\hat{s}^{2}-\hat{t}^{2}}{\hat{u}^{2}}-\frac{2}{3} \frac{\hat{s}^{2}}{\hat{u} \hat{t}} \\
\frac{\hat{s}^{2}-\hat{u}^{2}}{\hat{t}^{2}} \\
\frac{\hat{s}^{2}-\hat{u}^{2}}{\hat{t}^{2}}-\frac{\hat{u}^{2}+\hat{t}^{2}}{\hat{t}^{2}}+\frac{2}{3} \frac{\hat{u}^{2}}{\hat{s} \hat{t}} \\
-\frac{\hat{u}^{2}+\hat{t}^{2}}{\hat{s}^{2}} \\
\frac{\hat{s}^{2}-\hat{u}^{2}}{\hat{t}^{2}} \\
-\frac{8}{3} \frac{\hat{t}^{2}+\hat{u}^{2}}{\hat{t} \hat{u}}+6 \frac{\hat{t}^{2}+\hat{u}^{2}}{\hat{s}^{2}} \\
\quad-\frac{\hat{t}^{2}+\hat{u}^{2}}{\hat{t} \hat{u}}\end{array}$ & $\begin{array}{c}\frac{1}{\hat{s}^{2}} \\
\frac{2}{3 \hat{t} \hat{u}} \\
\frac{2}{\frac{s}{2}^{2}}-\frac{2}{3 \hat{t} \hat{t}} \\
\frac{{\frac{2}{\hat{s}^{2}}}^{-}}{\frac{16}{3 \hat{t} \hat{u}}-\frac{12}{\hat{s}^{2}}} \\
\frac{2}{\hat{u} \hat{t}}\end{array}$ \\
\hline
\end{tabular}
formalism of Gastmanns and $\mathrm{Wu}$ particularly well suited to these calculations. [G]

TABLE II. Parton cross sections and asymmetries. Each entry multiplies a factor of $\frac{4 \pi \alpha_{s}^{2}}{9 \hat{s}^{2}}$, except for the first (Drell-Yan) which multiplies $\frac{4 \pi \alpha_{e m}^{2} e_{q}^{2}}{3 \hat{s}^{2}}$, and the last (direct $\gamma$ production) which multiplies $\frac{8 \pi \alpha_{s} \alpha_{E M}}{9 \hat{s}^{2}}$. In addition, helicity entries multiply \pm 1 according to whether the beam helicities are equal $(+1)$ or opposite $(-1)$, and transversity entries multiply the kinematic factor $\left\{\hat{u} \hat{t} S_{a} \cdot S_{b}-\hat{s}\left(S_{a} \cdot k_{a} S_{b} \cdot k_{b}+S_{b} \cdot k_{a} S_{a} \cdot k_{b}\right)\right\}$, which is proportional to $\sin ^{2} \theta \cos 2 \phi$ in the parton-parton center of mass frame.

The same analysis applies to many hard processes. Another experimentally important example is high $p_{T}$ direct photon production, which proceeds via $q g \rightarrow q \gamma, \bar{q} g \rightarrow \bar{q} \gamma$, and $q \bar{q} \rightarrow g \gamma$ at lowest order in QCD. For longitudinal asymmetries all three processes contribute, but only the last survives for transverse asymmetries. The ratio of asymmetries is therefore

\footnotetext{
${ }^{1}$ Our values for $\sigma$ and $\delta \sigma$ differ slightly from those quoted by Ji for several subprocesses. [5] We
} are grateful to Dr. Ji for sharing his calculations with us. 


$$
\frac{\mathcal{A}_{T T}^{\gamma j}}{\mathcal{A}_{L L}^{\gamma j}}=\frac{[\delta q \otimes \delta \bar{q}] \delta \sigma_{q \bar{q}}^{\gamma j}}{[\Delta g \otimes \Delta q] \Delta \sigma_{g q}^{\gamma j}+[\Delta g \otimes \Delta \bar{q}] \Delta \sigma_{g \bar{q}}^{\gamma j}+[\Delta q \otimes \Delta \bar{q}] \Delta \sigma_{q \bar{q}}^{\gamma j}}
$$

The relevant parton cross sections are also given in Table II. Other hard processes for which selection rule $\mathcal{A}_{T T} / \mathcal{A}_{L L} \ll 1$ holds include

- $\vec{p} \vec{p} \rightarrow \pi+X$ - Inclusive production of a pion or other hadron at large transverse momentum requires inclusion of a $q \rightarrow \pi$ fragmentation function, which does not affect the argument given for two jet production.

- $\vec{p} \vec{p} \rightarrow Q \bar{Q}$ - Open heavy quark production is dominated by $g g$ fusion and therefore exhibits the same phenomena as $j j$ production.

Only Drell Yan production of charged lepton pairs through $q \bar{q} \rightarrow\left(Z^{0}, \gamma\right) \rightarrow \ell \bar{\ell}$ stands out as a case where transverse and longitudinal asymmetries are comparable. The ratio is given in terms of the ratio of quark transversity $(\delta q)$ and helicity $(\Delta q)$ distributions,

$$
\frac{\mathcal{A}_{T T}^{\bar{\ell}}}{\mathcal{A}_{L L}^{\bar{\ell}}}=-\frac{\sin ^{2} \theta \cos 2 \phi}{1+\cos ^{2} \theta} \frac{\sum_{a} e_{a}^{2} \delta q^{a}\left(x_{1}\right) \delta \bar{q}^{a}\left(x_{2}\right)+(1 \leftrightarrow 2)}{\sum_{a} e_{a}^{2} \Delta q^{a}\left(x_{1}\right) \Delta \bar{q}^{a}\left(x_{2}\right)+(1 \leftrightarrow 2)} .
$$

for $q \bar{q} \rightarrow \gamma^{*} \rightarrow \ell \bar{\ell}$. In contrast, Drell-Yan production of $\ell \bar{\nu}$ via $q \bar{q} \rightarrow W^{ \pm} \rightarrow \ell \bar{\nu}$ give no transverse asymmetry at leading twist. Only left handed quarks and antiquarks couple to $W^{ \pm}$so no helicity flip distribution functions participate at all. So for these processes

$$
\frac{\mathcal{A}_{T T}^{\ell \bar{\nu}}}{\mathcal{A}_{L L}^{\ell \bar{\nu}}}=0
$$

at leading twist to all orders in $\alpha_{Q C D}$. The first non-vanishing contribution appears at twistfour where $g_{T} \times \bar{g}_{T}$ can yield a chirality-even transverse asymmetry. Finally, before taking eq. (5) too seriously it is important to compute higher order corrections. If polarized gluons in the proton are copious then QCD corrections coming from processes like $g q \rightarrow q \gamma^{*} \rightarrow j \ell \bar{\ell}$ will contribute significantly to $\mathcal{A}_{L L}^{\bar{\ell}}$ but not to $\mathcal{A}_{T T}^{\bar{\ell}}$.

\section{RELATION TO EARLIER WORK}

The first suggestion that $\frac{\mathcal{A}_{T}^{j j}}{\mathcal{A}_{L L}^{j j}}$ should be small in QCD was made in a 1979 paper by Hidaka, Monsay and Sivers. [4] Their conclusions were quoted extensively in the 1983 review by Craigie, Hidaka, Jacob and Renard. [7] Their work is handicapped by the incomplete understanding of transverse spin in hard processes available at that time. In particular, gluons were ignored entirely and quark transversity distributions were described by the twist-three distribution function $g_{2}$. A $q g$ contribution to $\mathcal{A}_{T T}$ appears in the unnumbered equation following eq. (8) in Ref. [4] but is subsequently dropped without mention. Their treatment of transversely polarized quarks is incorrect. If transversely polarized quarks were described by $g_{2}$, then their effects would be suppressed in $q \bar{q} \rightarrow j j$ by a factor of $1 / s$ since $g_{T}$ is twist-three. Ref. [4] mistakenly treated the Wandzura-Wilczek contribution to $g_{T}$ as if it scaled. [8 Despite the errors in their argument, the conclusion that $\frac{\mathcal{A}_{T T}^{j j}}{\mathcal{A}_{L L}^{j j}}$ is very small remains correct. 
The publication of Ralston and Soper's 1979 paper introducing the twist-two transversity distribution made it possible to reconsider $\frac{\mathcal{A}_{T T}^{j j}}{\mathcal{A}_{L L}^{j j}}$ in the manner we have presented. 9 Recently many authors have considered transverse asymmetries in production and fragmentation processes at high momentum transfer. [5,2] The focus was on developing the proper interpretation of transverse polarization phenomena, on the information provided by measurements of transverse asymmetries and on the description of processes where the effects could be measured. Only Ji noted that transverse asymmetries in jet production are typically small and do not receive $g g$ contributions. [5] None of the papers pointed out the basis of the selection rule $\mathcal{A}_{T T} / \mathcal{A}_{L L} \ll 1$. Our purpose is to call attention to the fundamental importance of this result and to provide some numerical estimates of its magnitude.

\section{ESTIMATES FOR A POLARIZED COLLIDER}

The first collision of polarized protons at RHIC is scheduled for 1999. The beam energy will be variable and the center-of-mass energy will range $50<\sqrt{s}<500 \mathrm{GeV}$. The expected luminosity is $2.0(0.8) \times 10^{32} \mathrm{~cm}^{-2} \mathrm{sec}^{-1}$ at $\sqrt{s}=500(200) \mathrm{GeV}$. The polarization of the proton beam is expected to be $70 \%$. The most plausible integrated luminosity is $800(320) \mathrm{pb}^{-1}$ at $\sqrt{s}=500$ (200) GeV per year. In this Letter, we focus on the $p p$ collisions at $\sqrt{s}=500 \mathrm{GeV}$.

We have utilized the QCD event generator PYTHIA [10] for numerical studies of the spin asymmetries. We have calculated the asymmetries, $\mathcal{A}_{L L}$ and $\mathcal{A}_{T T}$, in various QCD processes with the polarized structure function sets obtained by Gehrmann and Stirling (GS95) [11] through next-to-leading order analysisf of the experimental data and the asymmetries calculated from the spin-dependent and spin-independent cross sections listed in Table 【1.

The polarized structure function set GS95 has three models of gluon polarization, all of which give equivalently good description of existing experimental data. The models GS95(A), (B), and (C) corresponds to large, medium, and small gluon polarization, respectively.

In our calculation, we have assumed that the transversity difference quark distribution $\delta q\left(x, Q^{2}\right)$ is identical to the helicity difference quark distribution $\Delta q\left(x, Q^{2}\right)$. There are several reasons for this choice. First, there is no data on $\delta q$ to guide us. Second, models typically find $\delta q \sim \Delta q$. The bag model predicts $\delta q$ of a shape and magnitude very similar to $\Delta q$; [12] a calculation by means of QCD sum rules suggests $\delta q$ which is smaller than $\Delta q$, but not by order of magnitude; [13] and preliminary results on the lattice calculation [14] of tensor charge which is the integrated value of quark transversity distributions, suggest $\delta \Sigma=0.64 \pm 0.12$. It should be noted that $Q^{2}$-evolution of $\delta q$ is completely different from $\Delta q$ so the assumption we have made cannot be valid for entire $Q^{2}$ region. In this situation the simplest assumption will allow further modification of the presented results rather easily when our knowledge of $\delta q$ improves.

\footnotetext{
${ }^{2}$ Their model includes the set obtained through leading order analysis. In our analysis only next-to-leading order version is employed.
} 


\section{A. Inclusive Jet Production}

All quark and gluon related tree level processes in the QCD have been simulated with PyтнiA. In the low $p_{T}$ region, gluon-gluon scattering dominates the cross section, and quark-quark contribution is dominant at high- $p_{T}$ region. Annihilation channels contribute only a very small fraction of order $10^{-3}$. No rapidity cut has been applied to the final jet in this calculation.

The asymmetries $\mathcal{A}_{L L}$ and $\mathcal{A}_{T T}$ for inclusive jet production have been calculated with GS95-models, (A), (B), and (C). The results are shown in Figure 2. The values of $\mathcal{A}_{T T}$ at $\phi=0$ are plotted for a comparison with $\mathcal{A}_{L L}$. The size of transverse asymmetry $\mathcal{A}_{T T}$ is about $5 \times 10^{-3}$ at $p_{T}=100 \mathrm{GeV} / c$. Such a small asymmetry will be hard to detect with RHIC detector systems with assumed integrated luminosity, so for practical purposes the QCD prediction is the "selection rule" $\mathcal{A}_{T T} / \mathcal{A}_{L L}=0$.

Clearly the longitudinal asymmetry $\mathcal{A}_{L L}$ for inclusive jet production is quite sensitive to the abundance of polarized gluons while $\mathcal{A}_{T T}$ is insensitive. We calculated $\frac{\mathcal{A}_{T T}}{\mathcal{A}_{L L}}$ for each $\Delta g$ model, and show the results in Figure 3 excepting the GS95(C) model, which gives an infinite value due to the zero-crossing in $\mathcal{A}_{L L}$.

It might be surprising that our $A_{L L}$ values are rather smaller than previous calculations [15]. Especially $q q$-scattering, which is the dominant process at high- $p_{T}$, contributes very little to the asymmetry. This is due to the cancellation of the asymmetry for uuscattering by the asymmetry for $u d$-scattering, since $\frac{\Delta d}{d}$ has opposite sign to $\frac{\Delta u}{u}$ and the partonic level asymmetry is larger for the scattering of different flavors than for the scattering of identical flavor. The strength of this cancellation could change significantly, if we take the model by Bourrely and Soffer [16], where flavor decomposition of polarized quark distribution is quite different from the GS models. This cancellation does not work on the $\mathcal{A}_{T T}$ since only the scattering of identical flavors contribute to the asymmetry.

\section{B. High- $p_{T}$ Direct Photon Production}

The transverse and longitudinal asymmetries for high- $p_{T}$ direct photon production are calculated in a similar way to the inclusive jet production. We have applied a cut on pseudo-rapidity, $|\eta|<0.35$ which is the acceptance of the electromagnetic calorimeter of PHENIX detector system at RHIC. The results are shown in Figure 1 Again, values at $\phi=0$ are plotted for $\mathcal{A}_{T T}$ to compare with $\mathcal{A}_{L L}$. The longitudinal asymmetries are order of a few percent, and are dependent on the assumed gluon polarization. The transverse asymmetries are at the $10^{-3}$ level. The ratio $\frac{\mathcal{A}_{T T}}{\mathcal{A}_{L L}}$ is calculated for each $\Delta g$ model, and plotted in Figure 5. The ratio ranges within $20 \%$ in the $p_{T}$ region where we expect significant statistics $(<30 \mathrm{GeV} / c)$ and again will be hard to detect experimentally at RHIC.

We conclude that the predicted transverse asymmetries in two jet and direct photon production are very small and, within the sensitivity of planned experiments, consistant with zero. Confirmation of $\mathcal{A}_{T T} / \mathcal{A}_{L L} \ll 1$ tests fundamental aspects of spin structure in perturbative QCD. Any nonzero asymmetry for the processes studied above, if observed, would require reconsideration of all spin effects in perturbative QCD. 


\section{ACKNOWLEDGEMENTS}

One of us (RLJ) would like to thank Xiangdong Ji for discussions, and the Physics Department at Harvard University for hospitality. We are grateful to Thomas Gehrmann for providing us a FORTRAN code to calculate the polarized structure functions. We would like to thank the organizers of the Adriatico Conference on Trends in Collider Spin Physics and the RIKEN Symposium on Spin Structure of the Nucleon where this collaboration was begun. A part of this work has been done within the framework of the RIKEN-BNL collaboration for the RHIC/Spin project. 


\section{REFERENCES}

[1] A. V. Efremov and O. V. Teryaev, Czech. Hadron Symposium (1988), 302; G. Alterelli and G. G. Ross, Phys. Lett. B212 (1988) 391; R. D. Carlitz, J. C. Collins and A. H. Mueller, Phys. Lett. B214 (1988) 229.

[2] For a review and references to the original literature, see R. L. Jaffe, "Spin, Twist and Hadron Structure in Deep Inelastic Processes, Lectures Presented at the 1995 Erice Summer School on the Spin Structure of the Nucleon", MIT Preprint MIT-CTP-2506, to be published in the proceedings, (January 1996) hep-ph 9602236.

[3] R. L. Jaffe and A. V. Manohar, Nucl. Phys. B321 (1989) 343; X. Artru and M. Mekhfi, Z. Phys. C45 (1990) 669. E. Sather and C. Schmidt Phys. Rev. D42 (1990) 1424.

[4] K. Hidaka, E. Monsay, D. Sivers, Phys. Rev. D19 (1979) 1503.

[5] X. Ji Phys. Lett. B284 (1992) 137.

[6] R. Gastmanns and T. T. Wu, The Ubiquitous Photon: The Helicity Method for QED and QCD (Clarnedon Press, Oxford, 1990).

[7] N. S. Craigie, K. Hidaka, M. Jacob, F. M. Renard, Phys. Rep. 99 (1983) 69.

[8] S. Wandzura and F. Wilczek, Phys. Lett. 72B (1977) 195.

[9] J. Ralston and D. E. Soper, Nucl. Phys. B152 (1979) 109.

[10] H.-U. Bengtsson and T. Sjötrand, Computer Physics Commun. 46 (1987) 43, CERNTH.6488/92 and references therein.

[11] T. Gehrmann and W. J. Stirling, Durham University preprint DTP-95-82 (1995), hep$\mathrm{ph} / 9512406$.

[12] R. L. Jaffe and X. Ji, Phys. Rev. Lett. 67 (1991) 552, Nucl. Phys. B375 (1992) 527.

[13] B. L. Ioffe and A. Khodzhamirian, Phys. Rev. D51 (1995) 3373; H. He and X. Ji, Phys. Rev. D52 (1995) 2960.

[14] T. Hatsuda, in Proceedings of the RIKEN symposium on "Spin Structure of the Nucleon", December 1995 (to be published.)

[15] C. Bourrely, J. P. H. Guillet, J. Soffer, Nucl. Phys. B361 (1991) 72.

[16] C. Bourrely and J. Soffer, Nucl. Phys. B445 (1995) 341. 


\section{Figures}
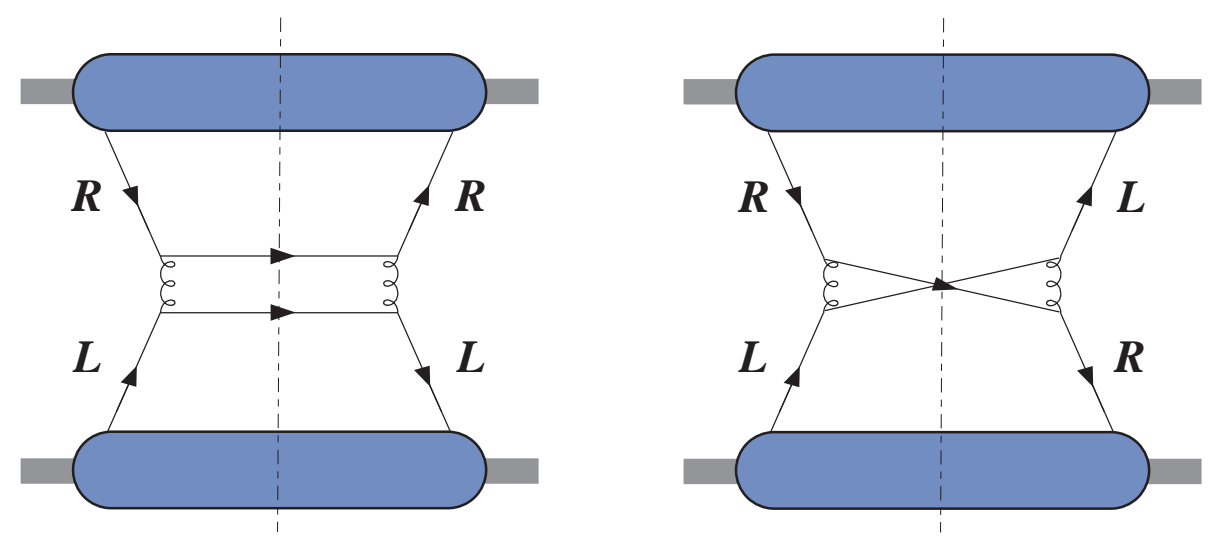

FIG. 1. Direct and Exchange graphs for $q q$ or $\bar{q} \bar{q}$ scattering.

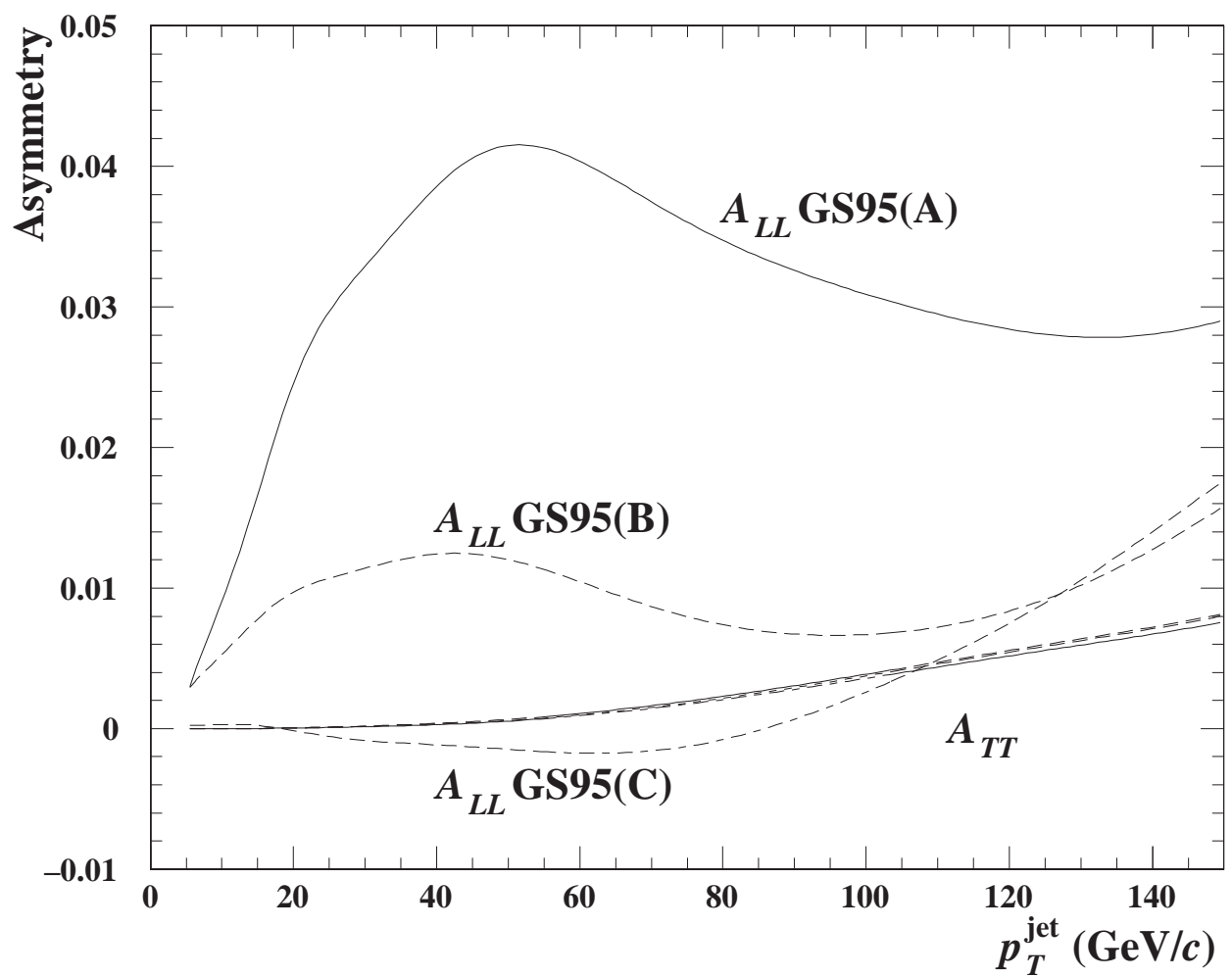

FIG. 2. Longitudinal and transverse asymmetries for inclusive jet production in $p p$-collisions at $\sqrt{s}=500 \mathrm{GeV}$. Asymmetries are calculated with three models of gluon polarizations, GS95(A) (solid), GS95(B) (dotted), and GS95(C) (dashed-dotted). 


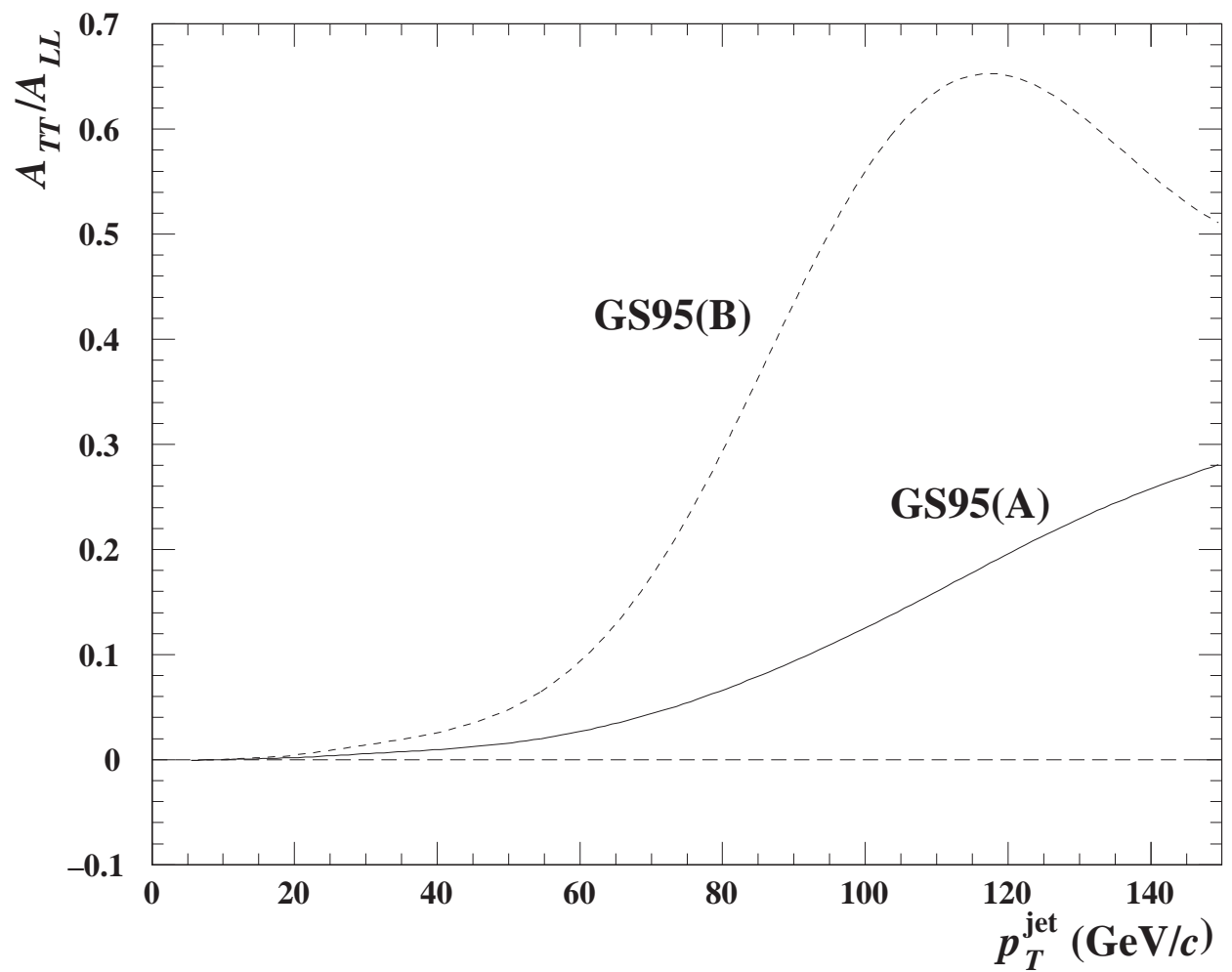

FIG. 3. Ratios of transverse asymmetries to longitudinal asymmetries for inclusive jet production in $p p$ collisions at $\sqrt{s}=500 \mathrm{GeV}$.

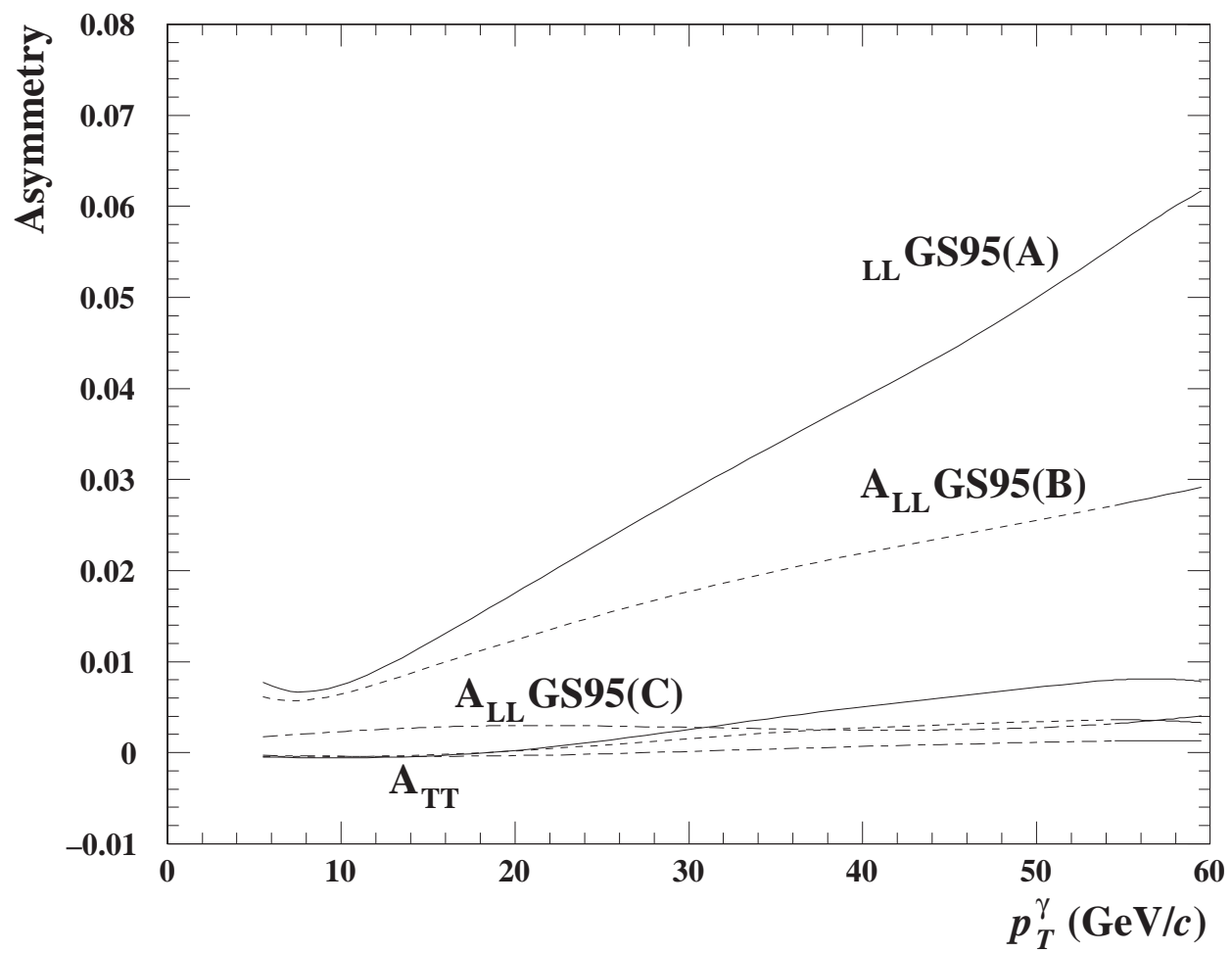

FIG. 4. Longitudinal and transverse asymmetries for high- $p_{T}$ direct photon production in $p p$-collisions at $\sqrt{s}=500 \mathrm{GeV}$. Asymmetries are calculated with three models of gluon polarizations, GS95(A) (solid), GS95(B) (dotted), and GS95(C) (dashed-dotted). 


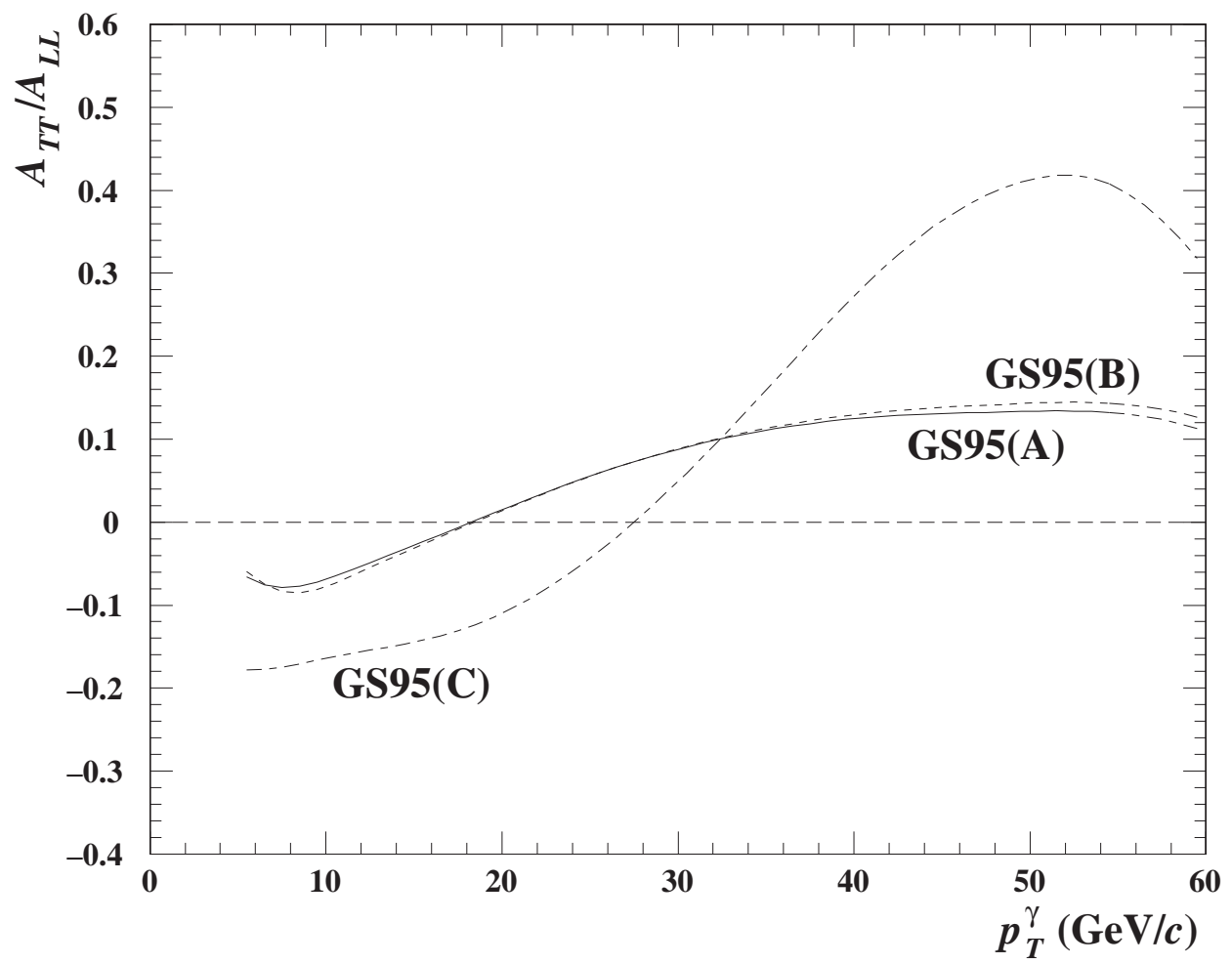

FIG. 5. Ratios of transverse asymmetries to longitudinal asymmetries for high- $p_{T}$ direct photon production in $p p$ collisions at $\sqrt{s}=500 \mathrm{GeV}$. 